\title{
ENABLING THIN SILICON TECHNOLOGIES FOR NEXT GENERATION C-Si SOLAR PV RENEWABLE ENERGY SYSTEMS USING SYNCHROTRON X-RAY MICRODIFFRACTION AS STRESS AND CRACK MECHANISM PROBE
}

\author{
A. S. BUDIMAN $^{1 *}$, G. ILLYA ${ }^{2}$, V. HANDARA ${ }^{2}$, W.A. CALDWELL ${ }^{3}$, \\ C. BONELLI ${ }^{4}, M$. KUNZ $^{5}, \mathrm{~N}$. TAMURA ${ }^{5}, \mathrm{D}$. VERSTRAETEN ${ }^{4}$ \\ ${ }^{1}$ Singapore University of Technology \& Design (SUTD), \\ Engineering Products Design (EPD) Pillar, \\ 20 Dover Drive, Singapore 138682; \\ Formerly of SunPower Corporation, R\&D, San Jose, CA 95134 \\ ${ }^{2}$ Center for Solar Photovoltaics Materials \& Technology (CPV), \\ Surya University, Boulevard Gading Serpong O/1, Summarecon Serpong, \\ Tangerang 15810, Indonesia \\ ${ }^{3}$ SunPower Corporation, R\&D, San Jose, CA 95134 \\ ${ }^{4}$ TOTAL Gas \& Power, R\&D Division, 92400 Courbevoie, France \\ ${ }^{5}$ Advanced Light Source (ALS), \\ Lawrence Berkeley National Laboratory (LBNL), Berkeley, CA 94720
}

\begin{abstract}
Recently, there has been a strong commercial push towards thinner silicon in the solar photovoltaic (PV) technologies due to the significant cost reduction associated with it. Tensile stress (normal, in-plane) and fracture of the silicon cells are increasingly observed and reported for products of crystalline solar cell technologies. In an effort to shed light on these topics, stress measurements and mapping of the solar cells in the vicinity of the most typically observed crack initiation locations using synchrotron X-ray microdiffraction technique was conducted and are reported in this paper. The technique is unique as it has the capabilities to quantitatively determine stresses in silicon and to map these stresses with a micron resolution, all while the silicon cells are already encapsulated.
\end{abstract}

1

(C) 2014. This manuscript version is made available under the Elsevier user license http://www.elsevier.com/open-access/userlicense/1.0/ 
With this technique, we aim to gain fundamental understanding of the stress magnitudes as well as characteristics that could lead to crack initiation and propagation. We have thus far found evidences of both extrinsic (device related) as well as intrinsic (crystallographic) nature of silicon cracking, which further confirm that the control of mechanical stress is the key to enable thin silicon solar cell technologies in the coming years. This study represents an ongoing high impact technology research that addresses real and important fundamental materials issue facing the crystalline silicon solar PV industry and contributes directly to the industry drive to reduce cost of $\mathrm{PV}$ systems to grid parity.

${ }^{*}$ Corresponding author: Arief S. Budiman, Singapore University of Technology and Design (SUTD); Email: suriadi@alumni.stanford.edu; Phone +65 64994503

KEYWORDS: Thin silicon; solar cell; synchrotron X-ray microdiffraction; stress; fracture.

\section{Introduction}

Silicon solar cell technology remains the most efficient and powerful way to commercially harness the sun's power for conversion to electricity. In order to keep improving the market adoption rate and thus providing solar energy that is costcompetitive with existing energy sources, the global silicon solar PV industry is aggressively reducing the cost of solar PV systems through innovations in materials and processes used in the manufacturing. One potential area of significant cost reduction is through the use of thinner silicon cells (<150 microns). However, coupled with other recent trends in the solar PV module technologies (thinner glass, lighter or no metal frames as well as increased use of some types of polymers for encapsulation of the cells), high stress and fracture of the silicon cells are 
increasingly observed and reported for products using thin silicon solar cell technologies. Cell cracks immediately lower module efficiency, and can lead to premature aging of the entire package/module.

Studying the fracture mechanisms in thin silicon solar cells has thus increasingly become both an interesting topic scientifically as well as an important subject technologically during recent years especially with the oncoming development of the new generations of solar PV renewable energy systems. More specifically, fractures in the thin silicon solar cells have been proposed to result from the high stress concentration area near the solder joints and busbars that provide interconnection between the cells [1-5]. Studying how stresses especially in these areas evolve during introduction of the solder joints in the solar cell assembly lines as well as later on during service and operational conditions/loading of the devices will be crucial in highlighting the propensity to fracture as well as its actual initiation and propagation mechanisms in the thin silicon solar cells. Crack propensity here is defined as the susceptibility of the material to initiate cracks and/or for the cracks to grow further. A material can be more susceptible to fracture when the stress level of the overall system is elevated due to the operational conditions or mechanical loading of the device/system. Therefore, the stress level in the material is approaching its fracture toughness which depends not only the strength of the materials but also the ubiquitous presence of crack-like defects and their size. Quantitatively, this happens when the stress intensity, $\boldsymbol{K}$, has approached the materials' critical stress intensity, $\boldsymbol{K}_{\boldsymbol{c}}$, or more generally, when the value of the strain energy release rate, $\boldsymbol{G}_{\text {, }}$ has approached the critical value of the strain energy release rate of the material, $\boldsymbol{G}_{\boldsymbol{c}}$. $\boldsymbol{K}_{\boldsymbol{c}}$ or $\boldsymbol{G}_{\boldsymbol{c}}$ here is a measure of fracture toughness which depends on both the strength of the material and its inherent flaw sizes [1].

However previous studies in the literature up to date on silicon cell fracture in C-Si solar PV technologies have been largely focused on the crack detection and imaging techniques [2, 3, 7-9]. 
Lacking the capacity to quantitatively characterize mechanical stress, which is the ultimate driving force of any crack initiation and propagation, these studies did not reveal much fundamental understanding of what affects the propensity to crack, the various failure modes, and, most importantly, the processes and loading conditions that lead to different states of mechanical stresses that ultimately cause different modes of the cracks. Synchrotron X-ray microdiffraction ( $\mu S X R D$ ) has thus proved to be a suitable technique to elucidate stress and crack propensity in stringed silicon solar cell assemblies. This technique provides quantitative determination of stresses (normal, hydrostatic and shear) in silicon as well as in solder joint material with a micrometer resolution [10-11]. It is non-destructive and the $\mathrm{X}$-ray beam has reasonable penetration depth which enables one to do in situ study of stresses in the silicon during cell operations or loading. This approach can thus provide us with the quantitative examination of stress and its evolution during the processes and operations of the devices in the realistic setting.

Stringed cell assemblies have always used solders and interconnects and they have always been the high stress concentration areas in the assemblies (Figure 1(a)). As the silicon cells get thinner (<150 microns), they acquire much higher sensitivities towards these high stresses and thus an increased propensity to cracks. Solders and metal interconnects induce high stresses in the silicon cells due to their typically very different coefficient of thermal expansion (CTE) values compared to that of silicon. Upon cooling from their typically high temperature introduction (solder reflow process), the CTE mismatch leads to high tensile in-plane stress in the silicon cell and this is what leads to crack initiation and propagation [3-5,12]. Most cell cracking observed in PV products from various companies as well as those reported in the literature $[2-5,12]$ are indeed initiated from the solder joint areas and typically propagate following the metallization lines. It is thus our main focus in this study to measure stress in the silicon cell around solder joint area after lamination and cooling processes, as well as to 
investigate further the source of these stresses, i.e. the solder joint itself.

The rest of the article is divided into three sections. Brief descriptions of the $\mu S X R D$ technique used in the present work and sample preparation needed are in the Experimental section. In the Results and Discussion section, we present and discuss the stress measurement in the silicon solar cell around a solder joint as well as the microstructure evolution of the solder joint materials comparing between as-reflowed solder joint materials and after thermal cycles and provide analysis how it could further aggravate the stress in the silicon near the solder joint area. The third section summarizes the significance of the findings and concludes the present study. 


\section{Experimental}

A solar PV module typically is a sandwich of a glass, a front encapsulant layer, a stringed cell assembly, a back encapsulant layer and finally a backsheet. However, in this study, we built only a mini module, which satisfied our purpose to study the stress in the silicon after lamination process. Our mini module consists of one $125 \mathrm{~mm}$ x $125 \mathrm{~mm}$ monocrystalline solar cell covered by encapsulant layers on both sides, a transparent backsheet layer at the back side, and a glass at the front side, laminated at high temperature using industry-typical processes and recipes (all materials are those typically used in the global PV industry) as shown in Figures 1 (b) (schematically, also showing how the in situ X-ray microdiffraction experiment was set up). Figure 1(c) shows the actual mini module sitting on the sample stage in the $\mathrm{X}$-ray microdiffraction experiment.

Stress measurements in the silicon around the solder joint were performed by synchrotron X-ray micro-diffraction at beamline 12.3.2 at the Advanced Light Source (ALS) of the Lawrence Berkeley National Laboratory [10]. The stress measurement in the silicon solar cell here is unique and novel as it is done while the solar cell is already laminated (encapsulated by transparent polymers and glass) thus representing the actual solar PV system in operations in the field. This approach thus allows in situ stress measurement of the silicon solar cell while the mini module is, for instance, being mechanically loaded or thermally cycled, to represent typical loading during real operations of the photovoltaic module system in the field. The polychromatic X-Ray beam produced from a superconductor magnet source is refocused at the entrance of the experimental hutch by a $700 \mathrm{~mm}-10 n g$ platinumcoated silicon toroidal mirror operating at a grazing angle of 4.5 mrad. Final focusing is done by Kirkpatrick-Baez mirrors consisting of an orthogonal pair of $100 \mathrm{~mm}-1$ ong tungsten-coated silicon substrate bent to an elliptical shape. The Synchrotron Xray micro-diffraction has 1 m x 1 um beam size, allowing one to obtain the local stress at any position on the sample. The sample sits on a XY high precision stage (range of +/- 10 microns), which 
is mounted on a coarse XYZ Huber stage (range of +/- $15 \mathrm{~mm}$ in XY and Z), see Figure $1(\mathrm{C})$. The diffraction patterns are collected with a DECTRIS Pilatus 1 M pixel area detector (active area of 179 x $169 \mathrm{~mm}$ ), which is placed at a distance of approximately $140 \mathrm{~mm}$ from the sample. The total strain tensor is typically obtained from the sum of the deviatoric components obtained by analyzing the Laue pattern and of the hydrostatic (dilatational) components obtained by energy scanning. The current setup in the beamline 12.3.2 provides the capabilities to do both measurements (deviatoric as well as hydrostatic stresses).

Additionally, in the present work, this technique was also used to study the microstructure of the solder joint materials after thermal cycling (ex situ). It is "ex situ" as we only compared here the X-ray diffraction patterns before vs. after the thermal cycling and not during the thermal cycling itself. Investigation of the microstructural evolution in the solder joint materials could lead to fundamental understanding of controlling the stress in the silicon solar cells, as solder joint is the stress concentrator causing the crack initiation. For this part of the study, only soldered cells without encapsulation material were investigated. The solder joint material used here is the typical tin solder materials with a few (atomic) percentage of silver (typically used in the solar PV industry) with typical grain sizes in the range of 0.5 - 15 microns. The solder joint connects the silicon solar cells to the busbar (tin coated cooper ribbon fig 2(c)), which in turns, connects one solar cell to another solar cell (Figure $2(\mathrm{~b})$ ). The thickness of the solder joint is a few tens of micrometers and its diameter is a few millimeters. The solder joint assembly was mounted in an epoxy and the sample was cut and polished right through the center of the solder joint to allow cross-sectional examination using synchrotron X-ray microdiffraction. The thermal cycling for this experiment was done separately for $2000 \mathrm{cycles}$, from $-40^{\circ} \mathrm{C}$ to $125^{\circ} \mathrm{C}$. 


\section{Results and Discussion}

Synchrotron $\mathrm{X}$-ray microdiffraction has been used to study and to map stress in single crystalline silicon wafer [11,13], however there are a few unique characteristics about the stress map in Figure 3. First of all, as shown in Figure 1, the silicon wafer here was already encapsulated by close to $0.5 \mathrm{~mm}$ of transparent polymers on each side. But with the high penetration of hard X-ray synchrotron radiation, we still could get the reflection from the silicon even though it was buried under $0.5 \mathrm{~mm}$ of encapsulant polymer material. This is a unique capability as it allows stress and microstructure evolution examination of silicon solar cells for instance during high temperature lamination process or even during the operation of the laminate, such as during thermal cycle or mechanical loading operation - a capability that has not hitherto been possible using charged particle microscopy techniques (Scanning Electron Microscopy and Transmission Electron Microscopy) or surface sensitive techniques (micro Raman spectroscopy) and could lead to the unraveling of potentially important and critical insights about what is really happening in the solar cell during lamination or what the deformation mechanisms are in the silicon solar cells during thermal cycling or mechanical loading. This unique capability has been recently highlighted in the Berkeley Lab Science Highlight for the work that we did on solar cells in the Advanced Light source (ALS) Berkeley Laboratory [14].

Secondly, the stresses here were actually determined not from the difference in lattice spacing before versus after the lamination process, but from the local curvature or, in other words, from the out-of-plane orientation of the Si (100) planes at any given pixel of the raster scanning. 3D crystal orientations of silicon were obtained from indexing the silicon Laue pattern. A methodology called "adaptive indexing" allows tracking the silicon reflections below the metallization layers thus enabling us to obtain the silicon 3D orientation information even after encapsulation. Curvature from all 3 directions (X, Y and Z) can thus be obtained. In that way, the stress analysis can be simplified and there is no 
need to determine all the stress components, including the hydrostatic part, which often can be time consuming. As it has been already known, the stress in the single crystal silicon could be determined from the local curvature of crystal using stoney's equation [15].

$$
\sigma_{S i}=\frac{Y_{m} t_{m}^{2}}{6 r t_{S i}\left(1-v_{m}\right)}=\frac{Y_{m} t_{m}^{2} \theta}{6 d t_{S i}\left(1-v_{m}\right)}
$$

Stoney's equation (1) is used to calculate residual stress in bent material, where $Y, t$ and $v$ are the Young's modulus, thickness, and Poisson's ratio, respectively, the subscripts $S i$ and $m$ denote silicon cell and metallization/solder joint, respectively [15]. The local radius of curvature $r$ is expressed as $r=d / \theta$, where $d$ is the distance from center to the edge of the silicon chip.

We essentially use the Stoney's equation here but due to the localized geometry of our measurement, we adopted some of the modifications and approximations as detailed in [15]. In principle, however, as shown in Equation (1), we could determine the stress in the material from how much it curves, or in other words, from the material's bending angle, $\theta$, while other parameters which are geometrical parameters of the sample/experiment as well as material properties are known.

This Stoney's approach (with some modifications and approximations) could lead to potentially unique capability in studying stress in silicon solar cells in situ during thermal cycling or mechanical loading or even during electrical/optical loading, which has not been hitherto possible or at least practical. By calculating how the curvature changes with the loading, the stress evolution could be determined almost in real time. This methodology could also help substantially in subtracting the diffraction pattern coming from the metallization and other layers on top of them, leaving behind only the diffraction pattern of the silicon substrate. 
As we can see in Figure 3(a), there are metallization patterns and very thick solder joint along with busbar (total thickness $~ 0.5$ $\mathrm{mm}$ ). The X-ray beam could not penetrate through the very thick solder joint and busbar and thus the dark region in the stress map indicating no information about silicon crystallography/stress was obtained from those pixels. But other than that, the X-ray beam was able to penetrate through the metallization layer and other materials, and thus we could obtain the silicon crystal stress everywhere else.

Our objective here is to measure the stress of the silicon in all area including the one right under the solder joint. The stress of silicon due to the solder joint could be calculated using thermal mismatch or could even be simulated using FEM (Finite Element Method), but the actual stress on the actual solar cell (especially the one that has been encapsulated within the PV device/system package) has never been measured experimentally. This manuscript reports on the measurement that we managed to obtain the stress of the silicon solar cell around the solder joint, but not right under the solder joint because of the limitation we have mentioned above. Although the stress right under the solder joint still needs to be determined, we now know the stress (the magnitude, the components, whether or not it is hydrostatic or deviatoric, etc.) right around the solder joint, and thus allowing us to design the solder joint accordingly in such a way to minimize fracture occurrence in the silicon solar cells.

It is evident from the stress mapping in Figure 3 that the stress state generally near the solder joint is in tensile (yellowish color) consistent with our expectation. The highest stresses seemed to be right around the solder joint (the red colored regions) amounting to about $300 \mathrm{MPa}$. The effect of the metallization lines could still be seen on the left and right of the lower part of the scanning. There are subtle horizontal patterns following the metallization lines there as we can see in Figure 3(a). This is also expected as metallization lines are where the stresses are expected to be higher due to CTE mismatch 
between silicon and the metal (i.e. Cu in this case). But overall, we can see the effect from the solder joint dominates and this is consistent with observations in the fields [3-5] as well as in the literature [12]. It was widely observed that majority of cracks originate from the solder joint areas indicating the highest stress area or stress concentration areas.

The X-ray scanning here was done in 10 microns step. The X-ray beam itself was approximately 1 micron in diameter. As a result, the data from a particular pixel is just from the area that is shined by $x$-ray. The 10 microns step size is sufficient as the metallization patterns in the solar cells do not have features that are typically smaller than 10 microns.

The micron resolution capability of the synchrotron X-ray microdiffraction allows the investigation of the local microstructure of the solder joint, which is a few tens of microns in thickness. Solder joint, as an interconnection between the solar cell and the environment, is usually designed to be compliant so that a large strain from the outside world, which can be caused by environments or unexpected mechanical loading, can be damped/absorbed. Thus, the stress on the solar cells can be minimized. When a solder joint forms a brittle layer, its overall stiffness increases significantly as the brittle phase tends to be stiffer or more rigid [17,18]. When a solder joint has effectively higher stiffness (thus lower compliance), it tends to transmit the stress more from the outside world to the solar cells increasing the stress and crack propensity inside silicon solar cell. Figure 4 shows the powder diffraction pattern of the solder joint material near the interface with $\mathrm{Cu}$ ribbon. The polycrystalline rings strongly indicate that due to the thermal cycling loading, a new intermetallic compound, $\mathrm{Cu}_{6} \mathrm{Sn}_{5}$, has formed as a result of activated interdiffusion between $\mathrm{Cu}$ and $\mathrm{Sn}$ (Figure 4(b)). This compound has been known to form whenever $\mathrm{Cu}$ and $\mathrm{Sn}$ are in contact and at elevated temperatures [16] $\mathrm{Cu}_{6} \mathrm{Sn}_{5}$ compound has also been known for its very brittle nature and thus could be particularly damaging and leading to higher stress and eventually producing fracture of the solder joint [16]. The presence of this 
layer/compound was not observed in the microstructure of the solder joint material in the bulk volume of the solder joint, far away from the interfaces with Cu ribbons (not shown in Figure 4).

This is an observation that would not have been possible with a conventional X-ray diffraction due to its limited resolution. The lack of resolution would only provide the microstructure of solder joint as an aggregate from all parts of the solder joint material. And if the intermetallic compound had been, for example, small volumes as they tend to be, then its existence might not have even been detected at all. Obviously, the small volumes of this very brittle compound would be enough to lead to catastrophic failure of the overall PV system by initiating cracks which would be propagating through the solder joint material and disconnecting the whole assembly. This is again the unique capability of the synchrotron X-ray microdiffraction technique to study and characterize stress and fracture in solar PV system.

\section{Conclusions}

Mechanical stress and fracture are the origins of failures of the solar PV cells during their fabrication as well as their operations in the fields. Thus it is very important to have the tools to characterize them in a more quantitative manner. Through the studies in this article, synchrotron x-ray microdiffraction has thus proved to be a very useful technique with unique capabilities to characterize stress quantitatively and investigate fracture mechanisms in solar cells as well as modules. Especially once the solar cells are laminated, this tool has proved to be perhaps the only practical technique that could provide the capability to study the stress in the silicon solar cells and how it evolves with fabrication processes (during lamination, cooling, etc.) as well as with operations or loadings in the fields. This has been an even more important capability as recently many researchers have started to observe the rather unexpected substantial effect of the mechanical properties of the encapsulant 
on the stress level and thus ultimately on the cracking rate of the silicon solar cells in the fully integrated PV package. If the effect of the polymer material is important then the silicon solar cell needs to be studied when it has already been encapsulated by it. Secondly, solder joint is the origin of majority of the silicon fracture. This technique provides the unique capability to study the local microstructure and how it evolves during processing or operations/loadings in the fields. This would enable, for instance, fundamental understanding of the origin and degradation mechanisms of the embrittlement of solder joint due to thermal cycling as shown in this manuscript. The exact understanding of the origin and the mechanisms of stresses in the thin silicon solar cells are the critical factors to enable the thin silicon (< 150 microns) technology for lower cost next generation PV systems.

\section{Acknowledgements}

ASB gratefully acknowledges research support and samples provided by SunPower Corporation. He also gratefully acknowledges critical support and infrastructure provided by Singapore University of Technology and Design (SUTD) during the writing of this manuscript. GI and VH gratefully acknowledge research support and infrastructure provided by Surya University through the Center for Solar Photovoltaics Materials and Technology (CPV; wWw.cpvsurya.com).

The Advanced Light Source is supported by the Director, office of Science, Office of Basic Energy Sciences, Materials Sciences Division, of the U.S.Department of Energy under Contract No. DE-AC02-05CH11231 at Lawrence Berkeley National Laboratory and University of California, Berkeley, California.

The move of the micro-diffraction program from ALS beamline 7.3.3 onto to the ALS superbend source 12.3.2 was enabled through the NSF grant \#0416243 


\section{References}

[1] M. E. Launey, R. O. Ritchie, On the Fracture toughness of advanced materials, Advanced Materials 21 (2009) 2103-2110.

[2] M. Sander, B. Henke, H. Schwarz, S. Dietrich, S. Schweizer, M. Ebert, J. Bagdahn, Characterization of PV modules by combining results of mechanical and electrical analysis methods, Proc. SPIE Reliability of Photovoltaic Cells, Modules and Systems III (2010) 777308 .

[3] J. Wendt, M. Träger, M. Mette, A. Pfennig, B. Jaeckel, The link between mechanical stress induced by soldering and micro damages in silicon solar cells, Proc. EUPVSEC (2009) 3420-3.

[4] A. Gabor, M. Ralli, S. Montminy, L. Alegria, C. Bordonaro, J. Woods, L. Felton, Soldering induced damage to thin Si solar cells and detection of cracked cells in modules, Proc. EUPVSEC (2006). [5] S. Nieland, M. Baehr, A. Boettger, A. Ostmann, H. Reichl, Advantages of microelectronic packaging for low temperature lead free soldering of thin solar cells, Proc. EUPVSEC (2007).

[6] K. Lin, Y. Lee, L. Wang, L. Chen, S. Yang, Electroluminescence observation of microcrack growth behavior of crystalline silicon solar modules fabricated by hot-air soldering technology, Advanced Materials Development and Performance (AMDP2011) International Journal of Modern Physics Conference Series 6 (2012) 643-48. [7] M. Demant, M. Glatthaar, J. Haunschild, S. Rein, Analysis of luminescence images applying pattern recognition techniques, Proc. EUPVSEC 1078 (2010) 82 .

[8] J. Haunschild, M. Glatthaar, M. Demant, J. Nievendick, M. Motzko, S.Rein, E. R. Weber, Quality control of as-cut multicrystalline silicon wafers using photoluminescence imaging for solar cell production, Solar Energy Materials and Solar Cells 94 (2010) 7-12.

[9] W. McMillan, T. Trupke, J.W. Weber, M. Wagner, U. Mareck, Y.C. Chou, J. Wong, In-line monitoring of electrical wafer quality using photoluminescence imaging, Proc. EUPVSEC 1346 (2010) 51. [10] N. Tamura, A. A. MacDowell, R. Spolenak, B. C. Valek, J. C. Bravman, W. L. Brown, R. S. Celestre, H. A. Padmore, B. W. Batterman and J. R. Patela, Scanning X-ray microdiffraction with 
submicrometer white beam for strain/stress and orientation mapping in thin films, J. Synchrotron Rad. 10137 (2003) 43.

[11] A.S. Budiman, H-A.-S. Shin, B.-J. Kim, S.-H. Hwang, H.-Y. Son, M.-S. Suh, Q.-H. Chung, K.-Y. Byun, N. Tamura, M. Kunz, Y.-C. Joo, Measurements of stresses in $\mathrm{Cu}$ and $\mathrm{Si}$ around Through-Silicon via (TSV) by synchrotron X-ray microdiffraction for 3-dimensional integrated circuits, Microelectron. Rel. 52 (2012) 530-533. [12] R. Meier ; M. Pander ; R. Klengel ; S. Dietrich ; S. Klengel ; M. Ebert ; J. Bagdahn, Reduction of soldering induced stresses in solar cells by microstructural optimization of copperribbons, Proc. SPIE. 8112 (2011) 811206-1.

[13] H.S. Shin, B.J. Kim, J.H. Kim, S.H. Hwang, A.S. Budiman, H.Y Son,K.Y. Byun, N. Tamura, M. Kunz, D.I. Kim, Y.C. Joo, Microstructure Evolution and Defect Formation in Cu ThroughSilicon Vias (TSVs) During Thermal Annealing, Journal of Electronic Materials 41 (2012) 712.

[14] A. S. Budiman, http://www-als.lbl.gov/index.php/sciencehighlights/industry-als/829-improving-thin-silicon-solar-celltechnology.html, 21 June 2013. Last accessed May 2014.

[15] K. Chen, N. Tamura, W. Tang, M. Kunz, Y.C. Chou, K.N. Tu, Y.S. Lai, High Precision Thermal Stress Study on Flip Chips by Synchrotron Polychromatic X-Ray Microdiffraction, Journal of Applied Physics 107 (2010) 063502.

[16] C. Chiu, K. Zeng, R. Stierman, D. Edwards, K. Ano, Effect of Thermal Aging on Board Level Drop Reliability for Pb-free BGA Packages, Electronic Components and Technology Conference Proceedings 54 (2004) 1256-1262.

[17] Z. Mei, M. Ahmed, M. Hu, G. Ramakhrisna, Kirkendall Voids at Cu/Solder Interface and Their Effects on Solder Joint Reliability, Electronic Components and Technology Conference Procedings, 2005, 415-420.

[18] B. J. Kim, G. T. Lim, J. Kim, K. Lee, Y. B. Park, H. Y. Lee, Y. C. Joo, Intermetallic Compound Growth and Reliability of Cu Pillar Bumps Under Current Stressing, Journal of Electronic Materials 39 (2010) 2281-2285. 
Figure 1. (a) Optical image of silicon cell around solder joint. (b) The incoming synchrotron focused $\mathrm{X}$-ray beam (the blue arrow) penetrates through the transparent backsheet layer (orange colored) and the back-side encapsulant layer (light green colored) and is diffracted by the silicon crystal (red arrows) allowing its crystallographic information to be captured by the CCD (ChargeCoupled Device) detector (black colored), which can then be translated into stress and, thus, crack propensity information. (c) The in situ experimental using synchrotron X-ray micro diffraction, where the encapsulated mini module sits on a XY piezoelectric sample stage tilted at 45 degree.

Figure 2. (a), (b) Two silicon cells with two solder joint interconnects. The red dots indicate the location of the solder joint material (the solder joint material is in between the silicon solar cell on the bottom and the busbar on the top). (c) An SEM image taken at the cross-section A-A as shown in (a) of the solder joint assembly which is embedded in the epoxy mould. The solder joint material is denoted by (i), where the one on top (ii) is solder material residue of the soldering process. The brown part is the busbar material (iii) and the flat part (iv) is the silicon solar cell.

Figure 3. (a) Optical image of a silicon solar cell around a solder joint of the mini module sample as illustrated in Figure 1 (b) and 1(c). (b) Local curvature map of encapsulated silicon cell surrounding the solder joint (corresponding to the same area in (a). Silicon under the solder joint (dark color in the map) is not mapped due to the $\mathrm{x}$-ray's inability to penetrate through the thickness of solder joint (which is a few tens of microns). 
Figure 4. (a) The powder diffraction pattern of the solder joint material obtained from X-ray microdiffraction resulting in the polycrystalline rings. (b) The enlargement of part (a) which is marked by red border lines; the indices, according to cu6sn5 crystal, which fit perfectly with the polycrystalline rings observed in our experiment, indicate the present of $\mathrm{Cu}_{6} \mathrm{Sn}_{5}$ intermetallic compounds, amongst others, which were not present in the sample before the thermal cycles. 


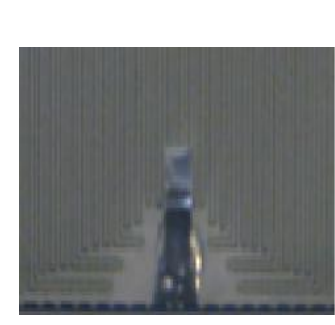

(a)

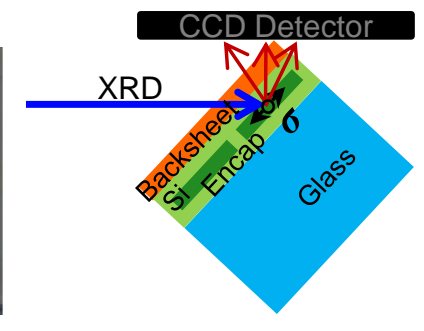

(b)

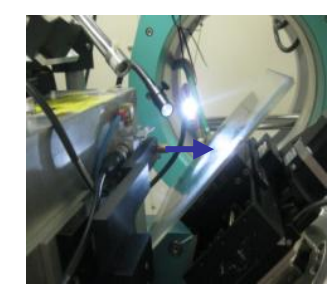

(c) 


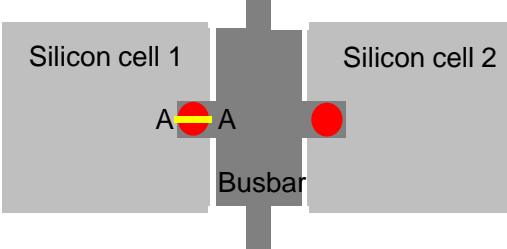

(a)

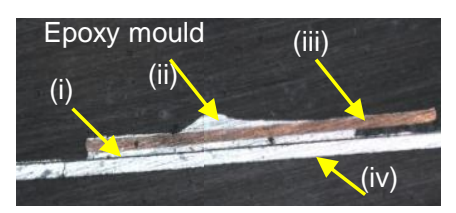

(b) 


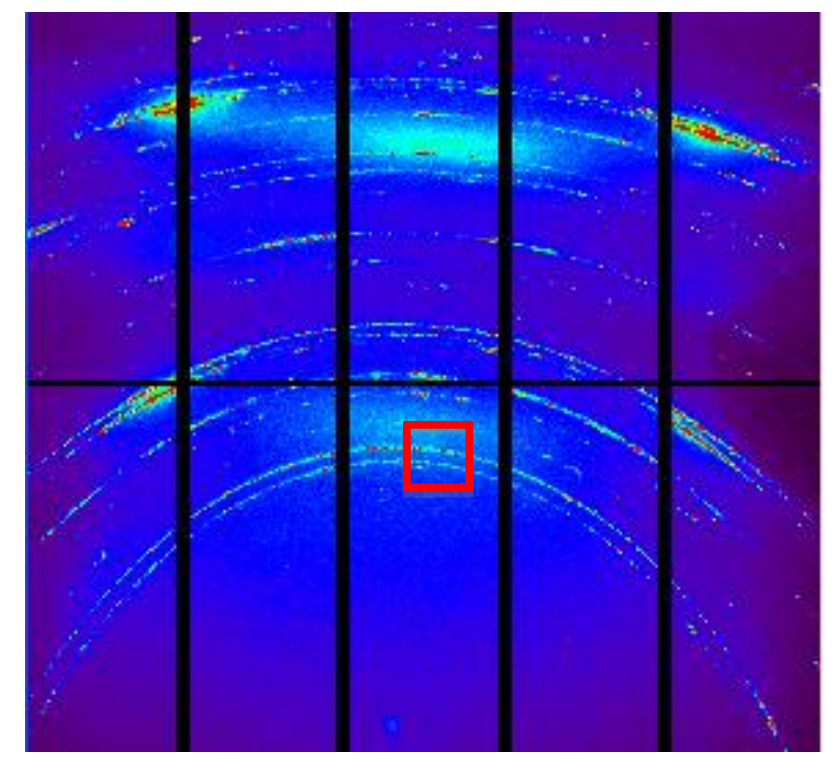

(a)

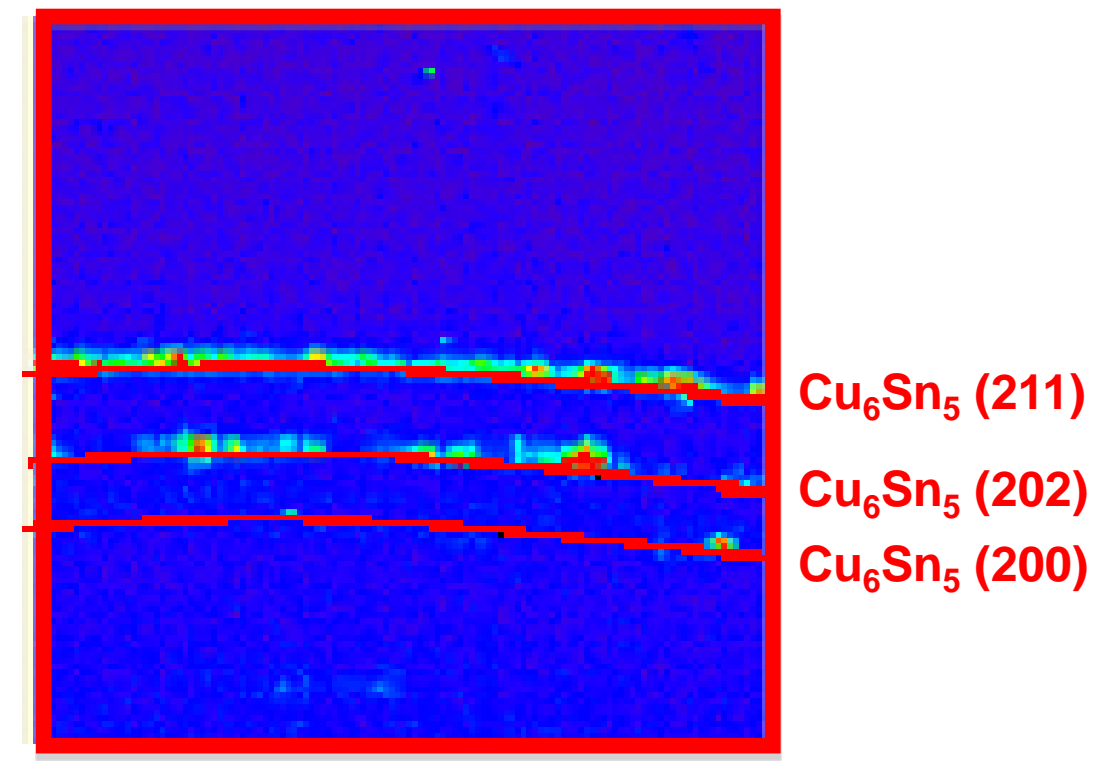

(b) 